EPJ Web of Conferences 38, 17006 (2012)

DOI: $10.1051 /$ epjconf/20123817006

(C) Owned by the authors, published by EDP Sciences, 2012

\title{
Origins of dipole resonance strength fragmentation in calcium and titanium isotopes
}

\author{
N. G. Goncharova and Iu. A. Skorodumina \\ Skobeltsyn Institute for Nuclear Physics, Moscow State University, Moscow, 199992, Russia
}

\begin{abstract}
Theoretical description of dipole resonances in ${ }^{46} \mathrm{Ti},{ }^{48} \mathrm{Ti},{ }^{50} \mathrm{Ti},{ }^{48} \mathrm{Ca},{ }^{40} \mathrm{Ca}$ was performed. The distribution of the "hole" among the states of final nuclei was taken into account using information on pick-up reactions. The obtained results are in reasonable agreement with experimental data.
\end{abstract}

\section{Description of multipole resonances}

The giant multipole resonances (MR) represent the most striking feature of the reactions cross sections up to energy excitation $\mathrm{E}<40 \mathrm{MeV}$ and transferred momentum $\mathrm{q}<3 \mathrm{fm}^{-1}$. The interpretation of MR complicated structure and its dependence on the individual properties of nuclei is one of the aims of nuclear theory. The giant dipole resonance E1 is up to now the best explored among the multipole resonances [1]. The efforts to explain the origin of its position on energy axis promote the creation of multiparticle shell model (MSM). The success of MSM in interpretation of dipole resonance as a result of collectivization of so-called "doorway states" could not eliminate all problems in its theoretical description. One of them is the need to explain the great differences in widths and structures of the distributions of E1 strength in various nuclei. The growth of information on structure of giant resonances has shown that the MSM calculations based on particle-hole configurations are unable to reproduce complicated structure of MR. The usual way to overcome this problem is to expand the basic space and to take into account the interaction of "doorway" states with more complicated configurations, first of all with collective phonons. Applications of this method to the resonances in the middle and heavy closed-shell nuclei were rather successful, but the interpretation of structure and decay properties of MR in open shell nuclei represents a challenge to the theory. Moreover, due to pairing forces the "magic" nuclei are not bona fide completely closed-shell systems.

\section{Particle-core coupling version of Shell Model}

One of the possible ways to build a set of basic configurations which could be used as doorway states in the microscopic description of multipole resonances in open shell nuclei is to take into account the distribution of the "hole" configurations among the states of residual (A-1) nuclei.

In the "Particle Core Coupling version of Shell Model" (PCC SM) these distributions are taken into account in microscopic description of multipole resonances $[2,3]$. Theoretical description of MR in 1pshell nuclei in the PCC SM has shown good agreement with experimental data for nuclei with A from 7 up to 15 [3]. The same approach to sd-shell nuclei strikes against the lack of reliable wave functions for the nuclear ground states. The alternative way to estimate the probabilities of the various core states which appears when one of the nucleons would be extracted from nucleus is to use the experimental data on the spectroscopy of direct pick-up reactions .This method was applied to calculations of multipole resonances in sd-shell nuclei [4]. Wave functions of excited nuclear states in the PCC SM approach are expanded to a set of low-lying states of (A1) nuclei coupled with a nucleon in a free orbit

$\left|J_{f} T_{f}\right\rangle=\sum_{\left(J^{\prime}\right), j^{\prime}} \alpha_{f}^{\left(J^{\prime}\right), j^{\prime}}\left|\left(J^{\prime} E^{\prime} T^{\prime}\right)_{A-1} \times\left(n^{\prime} l^{\prime} j^{\prime}\right): J_{f} T_{f}\right\rangle$

The basic configurations for MR in nuclei under investigation should be built on those states of residual (A-1) nuclei which have non-vanishing coefficients of fractional parentage $C$ in the expanding of ground state wave function of target nucleus:

$\left|J_{i} T_{i}\right\rangle=\sum_{\left(J^{\prime}\right), j^{\prime}} C_{i}^{\left(J^{\prime}\right), j^{\prime}}\left|\left(J^{\prime} E^{\prime} T^{\prime}\right)_{A-1} \times(n l j): J_{i} T_{i}\right\rangle$

The coefficients in the set (2) were estimated as

$$
C_{i}=S_{i} / \sum_{k} S_{k}
$$

where $S_{\mathrm{i}}$ are the spectroscopic factors of pick-up reactions. Matrix elements of the PCC Hamiltonian 
involve the excitation energies of the levels of final nuclei

$\widehat{H}_{i j}=\left(E^{\prime}+\varepsilon_{j}+E_{c}\right) \delta_{i j}+\widehat{V}_{i j}$

The estimation of residual interaction matrix elements was based as well on probabilities of pick-up reactions and corresponds to the following scheme:

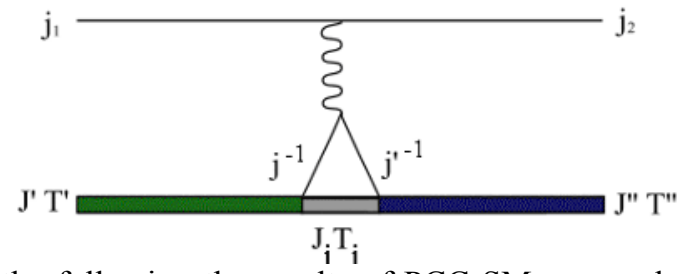

In the following the results of PCC SM approach to the dipole resonances in even-even isotopes of titanium and calcium will be discussed.

Diagonalization of the Hamiltonian (4) on the set of basic configurations produces energies and wave functions of excited states. The single-particle energies $\varepsilon_{\mathrm{j}}$ of nucleons removed via $1 \mathrm{~h} \omega$ transitions were calculated in the Saxon-Woods potential well.

\section{Results of PCC SM calculations for ${ }^{46} \mathrm{Ti},{ }^{48} \mathrm{Ti}$ and ${ }^{50} \mathrm{Ti}$}

In the sets of basic configurations for the E1 excitations all the states of (A-1) nucleus which have noticeable spectroscopic factors $(S>0.01)$ of direct reaction for neutron pick-up were included. For isotopes ${ }^{46} \mathrm{Ti},{ }^{48} \mathrm{Ti}$ and ${ }^{50} \mathrm{Ti}$ these $S$ factors were obtained from (p, d) reaction data [5]. For ${ }^{40} \mathrm{Ca}$ and ${ }^{48} \mathrm{Ca}$ the $S$ factors were extracted from [6] and [7], respectively. Wave functions for $1^{-} T_{<}$and for $1^{-} T_{>}$states in $\mathrm{Ca}$ and $\mathrm{Ti}$ even-even isotopes were used to calculate the $E 1$ form factors at photopoint.

Calculations of form factors and partial cross sections for photodisintegration were made with specially constructed code to diagonalize the full Hamiltonian matrices and estimate the partial width peaks. The widths were estimated applying the R-matrix method.

The PCC SM calculations of the dipole resonance in the $\mathrm{Ca}$ and $\mathrm{Ti}$ even-even isotopes show that $E 1$ strength is highly fragmented due to distribution of states in a final nucleus over excitation energy. The structure of isovector dipole states in ${ }^{46} \mathrm{Ti},{ }^{48} \mathrm{Ti},{ }^{50} \mathrm{~T}$ and ${ }^{48} \mathrm{Ca}$ nuclei is influenced as well by isospin splitting of $\mathrm{T}_{<}$and $\mathrm{T}_{>}$states. An example of isospin factors working upon excitation and decay of isovector resonance is shown in figure 1 for ${ }^{46} \mathrm{Ti}$.

The results of PCC SM calculations for the $(\gamma, n)$ reactions on titanium isotopes are shown in figures 2-4 together with experimental data (the right axis in the figures). The solid lines show the results of calculations of summed probabilities based on the estimations of peak widths.

Since photodisintegration probabilities depend on the structure of wave functions and isospin of final nuclear states, the ratio between photoproton and photoneutron channels changes from peak to peak.

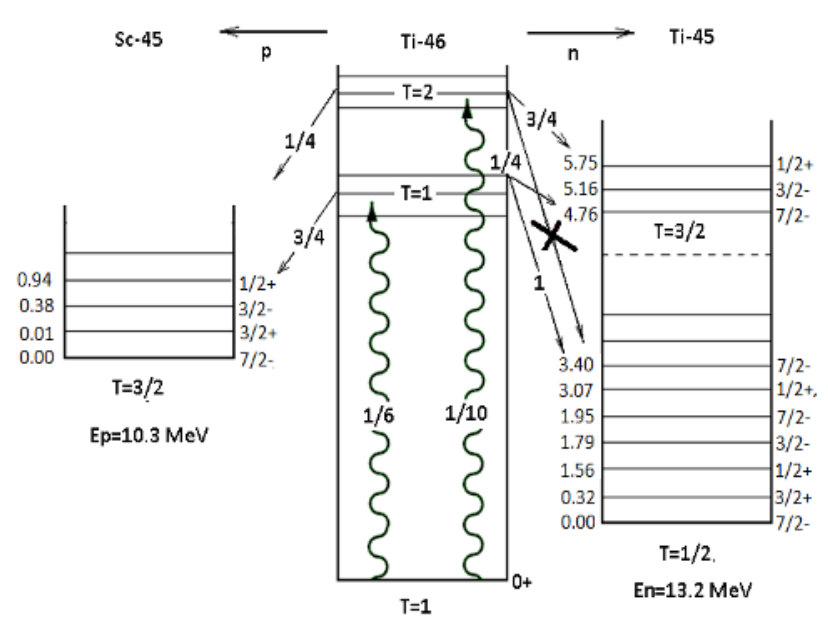

Fig.1. Squared isospin coefficients for excitation and decay of ${ }^{46} \mathrm{Ti}$.

In figures $2 \mathrm{a}-\mathrm{b}$ are shown the calculated form factors for photoexcitation of ${ }^{46} \mathrm{Ti}$. The differences in the structure of $1^{-}$peaks leads to different probabilities of nucleon decay channels. For example, the peaks at $\mathrm{E} \approx 18 \mathrm{MeV}(\mathrm{T}=1)$ are built mostly on the $\mathrm{T}=3 / 2$ states of final nuclei with $\mathrm{A}=$ 45 .

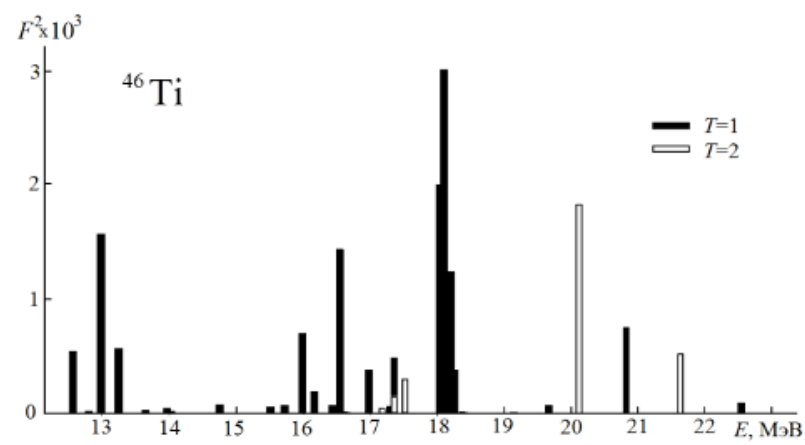

Fig.2a. Distributions of $E 1$ form factors in ${ }^{46} \mathrm{Ti}$.

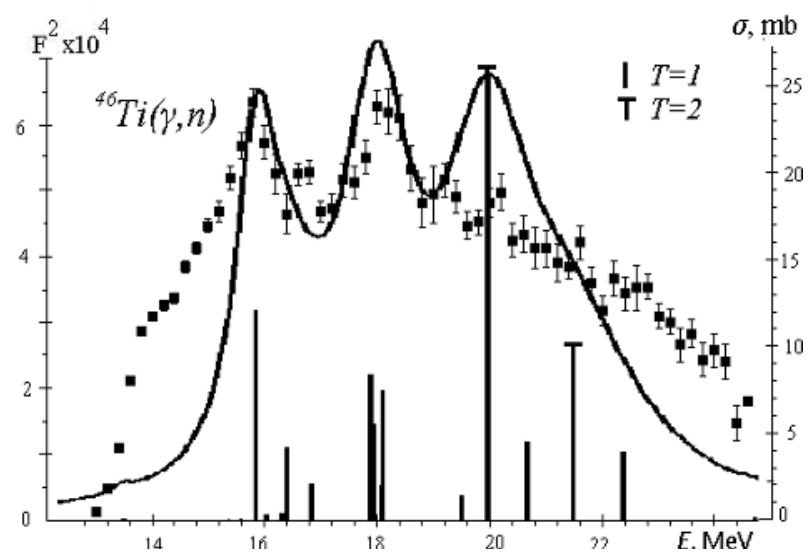

Fig. 2b. ${ }^{46} \mathrm{Ti}(\gamma, n)$. Experimental data are from [8].

As a consequence, they decay mainly through proton emitting. For the $\mathrm{T}=2$ peaks at 20 and $21.6 \mathrm{MeV}$, on the contrary, $(\gamma, \mathrm{n})$ channel is about 3 times more probable than $(\gamma, p)$. 


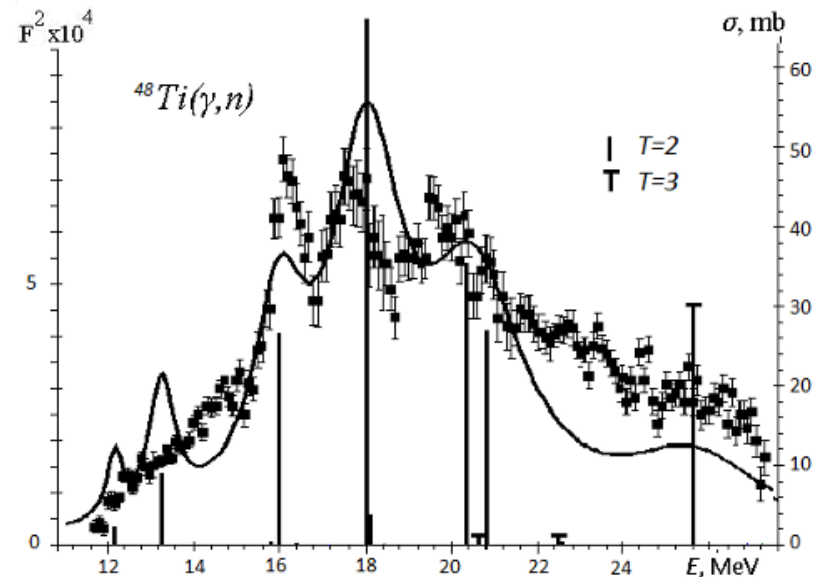

Fig. 3. $E 1$ for ${ }^{48} \mathrm{Ti}(\gamma, n)$. Experimental data are from [9].

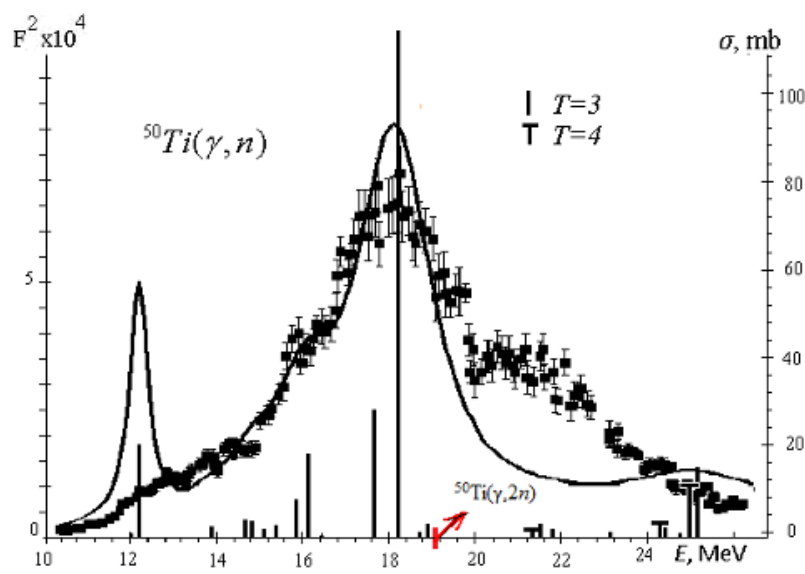

Fig.4. $E 1$ for ${ }^{50} \mathrm{Ti}(\gamma, n)$. Experimental data are from [10].

\section{Results of PCC SM calculations for ${ }^{40} \mathrm{Ca}$ and ${ }^{48} \mathrm{Ca}$}

The giant dipole resonance in the double-magic ${ }^{40} \mathrm{Ca}$ belongs to the most detailed investigated ones. Our results are displayed in figure 5. In the PCC SM calculations we used spectroscopic factors from [6]. According to the wave functions structure in the PCC SM approach, the bump in ${ }^{40} \mathrm{Ca}(\gamma, \mathrm{n})$ at $E_{\gamma}=23-25 \mathrm{MeV}$ corresponds to contributions of basic configurations built on $5 / 2^{+}$states of the final nucleus with $A=39$. The wide energy distribution of these states revealed in ${ }^{40} \mathrm{Ca}(\mathrm{d}, \mathrm{t})$ reaction leads to a spreading of resonance strength.

The PCC SM results for $E 1$ resonance in ${ }^{48} \mathrm{Ca}$ are displayed in figure 6. The spectroscopic factors used in the calculation are taken from ${ }^{48} \mathrm{Ca}(\mathrm{d}, \mathrm{t}),{ }^{48} \mathrm{Ca}(\mathrm{p}, \mathrm{d})$ reactions [7] where projectile energies were not higher than $40 \mathrm{MeV}$. The comparison of two direct experiments [6] and [7] shows that if the energy of projectile is low, the sum of spectroscopic factors for subshell would be underestimated, e.g. the summed occupation number for $1 d_{5 / 2}$ subshell in ${ }^{48} \mathrm{Ca}$ according to [7] is only 0.95 . The same number for ${ }^{40} \mathrm{Ca}$ is 5.41 (see [6]). Therefore the calculation of $E 1$ distribution in ${ }^{48} \mathrm{Ca}$ was performed with corrected occupation numbers for $\mathrm{d}_{5 / 2}$.

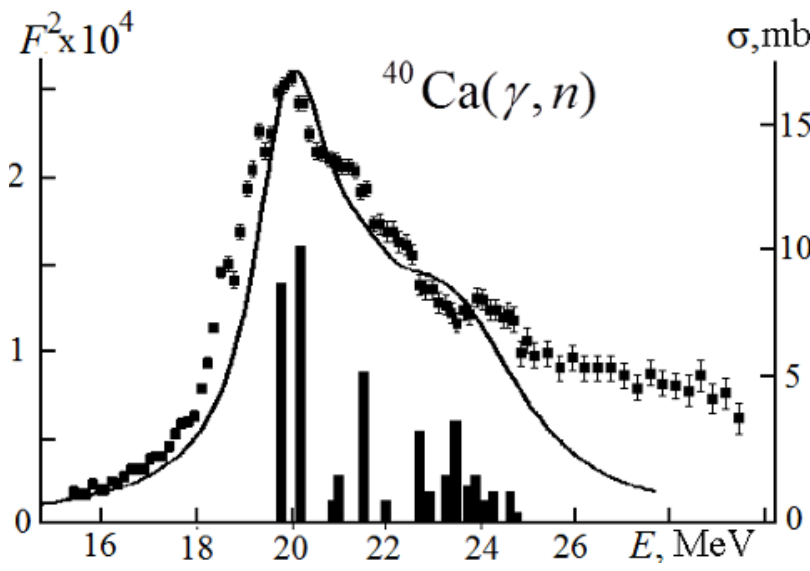

Fig. 5. Results of calculations for ${ }^{40} \mathrm{Ca}(\gamma, \mathrm{n})$ and experimental data [11].

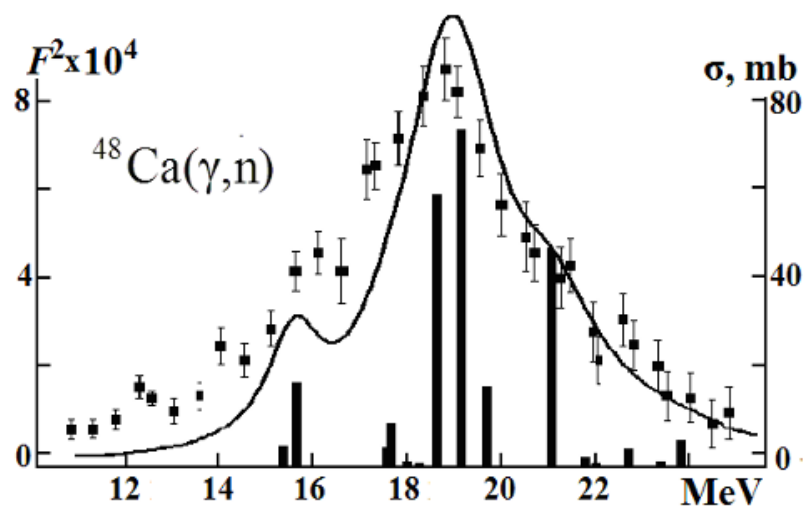

Fig. 6. Calculated and experimental [12] $E 1$ distributions in ${ }^{48} \mathrm{Ca}(\gamma, n)$.

The analysis of the isospin branches in non-self conjugated titanium isotopes and in ${ }^{48} \mathrm{Ca}$ presented in Table shows that obtained mean distance between $\mathrm{T}_{<}$and $\mathrm{T}_{>}$states is in the frame of estimations [13].

Table 1. Mean energies for $T_{<}$and $T_{>}$branches and the isospin splitting.

\begin{tabular}{|c|c|c|c|c|}
\hline$E, \mathrm{MeV}$ & ${ }^{46} \mathrm{Ti}$ & ${ }^{48} \mathrm{Ti}$ & ${ }^{50} \mathrm{Ti}$ & ${ }^{48} \mathrm{Ca}$ \\
\hline$T$ & 1 & 2 & 3 & 4 \\
\hline$E\left(T_{<}\right)$, & 17.7 & 18.1 & 18.7 & 20.5 \\
\hline$E\left(T_{>}\right)$, & 20.0 & 23.6 & 25 & 26.8 \\
\hline$\Delta E$ & 2.3 & 5.5 & 6.3 & 6.3 \\
\hline$\Delta E=60\left(T_{0}+1\right) / A$ & 2.6 & 3.75 & 4.8 & 6.25 \\
\hline$\Delta E=100\left(T_{0}+1\right) / A$ & 4.3 & 6.25 & 8 & 10.4 \\
\hline
\end{tabular}




\section{Conclusions}

First, calculations of the $E 1$ strengths in calcium and titanium isotopes shown that one of the main reasons of resonance strength fragmentation is the energy spread of single-particle strength in final nuclei. This factor is taken into account in the PCC version of the shell model. Second, for the realistic description of the nuclei disintegration due to interactions with electromagnetic fields the spectroscopic information on direct reactions have to be obtained with energy of projectiles by 15 or more $\mathrm{MeV}$ higher than the maximal photon energy.

\section{References}

1. R. Eramzhyan, B. Ishkhanov et al., Phys. Rep. 136, 229 (1986)

2. N. Goncharova, N. Yudin, Phys. Lett. B 29, 272 (1969)

3. N. Goncharova, et al., Sov. J. Part. Nucl. 16, 337

(1985)

4. N. Goncharova, Phys. At. Nucl. 72, 1803 (2009)

5. P. Plauger, E. Kashy, Nucl.Phys. A 152, 609 (1970)

6. P. Doll, et al., Nucl. Phys. A 263, 210 (1976)

7. T. Burrows, Nucl. Data Sheets, 108, 923 (2007)

8. P. Pywell, M. Thompson et al., Nucl. Phys. A 318, 461 (1979)

9. R. Sutton, M. Thompson et al., Nucl. Phys. A 339, 125 (1980)

10. P. Pywell, M. Thompson et al., Nucl.Phys. A 325,

116 (1979)

11. A. Veyssiere, et al., Nucl. Phys. A 227, 513 (1972)

12. G. O'Keefe, M. Thompson et al., Nucl. Phys. A 469, 239 (1987)

13. A. Lane, Nucl. Phys. 35, 676 (1962) 